\section{BMJ Paediatrics Open}

\title{
Rapid systematic review of neonatal COVID-19 including a case of presumed vertical transmission
}

Morris Gordon (D) ,',2 Taher Kagalwala (D) ,' Karim Rezk, ${ }^{2}$ Chris Rawlingson, ${ }^{2}$ M Idris Ahmed, ${ }^{2}$ Achyut Guleri ${ }^{3}$

To cite: Gordon M, Kagalwala T, Rezk K, et al. Rapid systematic review of neonatal COVID-19 including a case of presumed vertical transmission. BMJ Paediatrics Open 2020;4:e000718. doi:10.1136/ bmjpo-2020-000718

Received 28 April 2020 Revised 13 May 2020 Accepted 16 May 2020
Check for updates

\section{(C) Author(s) (or their} employer(s)) 2020. Re-use permitted under CC BY-NC. No commercial re-use. See rights and permissions. Published by BMJ.

${ }^{1}$ Biomedical evidence synthesis and translation to practice unit, University of Central Lancashire, Preston, UK

${ }^{2}$ Families division, Blackpool Victoria Hospital, Blackpool, UK ${ }^{3}$ micrbiology department, Blackpool Victoria Hospital, Blackpool, UK

Correspondence to Dr Morris Gordon; Mgordon@ uclan.ac.uk

\section{ABSTRACT}

Objective To carry out a systematic review of the available studies on COVID-19 (coronavirus disease 2019) in neonates seen globally since the onset of the COVID-19 global pandemic in 2020. The paper also describes a premature baby with reverse transcription (RT)-PCRpositive COVID-19 seen at the Blackpool Teaching Hospitals NHS Foundation Trust, UK.

Design We conducted a multifaceted search of the Cumulative Index to Nursing and Allied Health Literature, Embase, Medline and PubMed from 1 December 2019 to 12 May 2020 to harvest articles from medical journals and publications reporting cases of COVID-19 in neonates from anywhere in the world. Additional searches were also done so as not to miss any important publications. Write-up was in line with the Preferred Reporting Items for Systematic Reviews and Meta-Analyses, the protocol for the review was registered with International Prospective Register of Systematic Reviews (PROSPER0), and risk of bias was analysed with the Newcastle-Ottawa tool. Additionally, the preterm neonate with COVID-19 from our hospital is also reported.

Results The systematic review has revealed eight studies where neonates have been described to have confirmed COVID-19, with low risk of bias. Of the 10 reported cases elsewhere, only three are likely to be vertically transmitted, while seven occurred in the postperinatal period and are likely to have been postnatally acquired. All neonates had a mild course, recovered fully and were negative on retesting. Our case of COVID-19 in a 32-week premature baby from the UK was delivered by emergency caesarean section, with the mother wearing a face mask and the family having no contact with the neonate, suggesting vertical transmission. On day 33 , the neonate was asymptomatic but was still RT-PCR-positive on nasopharyngeal airway swab.

Conclusions Neonatal infection is uncommon, with only two previously reported cases likely to be of vertical transmission. The case we report is still RT-PCR-positive on day 28 and is asymptomatic. Ongoing research is needed to ascertain the epidemiology of COVID-19 in neonates.

\section{INTRODUCTION}

The novel coronavirus disease (COVID19) is a highly contagious disease that was first reported in Wuhan, Hubei Province, China in December 2019. Within weeks of
What is known about the subject?

COVID-19 has been described globally in children and adults of all age groups.

- Few cases have been reported in neonates and preterm babies.

\section{What this study adds?}

This review found only 10 published cases of COVID-19 in neonates, as well as reporting a further case.

- The course appears mild and vertical transmission even rarer, occurring in only three cases.

the emergence of the disease, it has spread to several countries, and the WHO declared the outbreak as a public health emergency of international concern in January 2020 and as a pandemic in March 2020. ${ }^{1}$ According to the WHO's Situation Report 113 published on 12 May 2020, coronavirus now affects 210 countries and 2 international conveyances, and has affected more than 4100000 persons, with more than 283000 individuals dead. ${ }^{2}$

COVID-19 is caused by severe acute respiratory syndrome coronavirus 2 (SARS-CoV-2), previously known as the 2019 novel coronavirus or 2019-nCoV. SARS-CoV-2 is a novel member of coronaviruses, which are a large class of highly diverse, enveloped, positivesense, single-stranded RNA viruses. ${ }^{3}$ Most reported cases of the disease are in adults, but the disease has also been reported in children, including neonates. ${ }^{4}$

While COVID-19 typically presents as acute respiratory disease and pneumonia, it has been reported to also impact other systems including the gastrointestinal tract. ${ }^{5}$ The first reported paediatric case of COVID-19 was probably of a 10-year-old boy from Shenzhen, China diagnosed with the condition in 
January $2020{ }^{6}$ Since then, there have been a few other reports of confirmed COVID-19 in neonates.

We did a rapid systematic review to summarise the published data on neonatal presentations of COVID-19. We also report a case of a 32-week premature baby with COVID-19 born to a COVID-19-affected mother.

\section{METHODS}

\section{Search strategy and study selection}

This study is reported in accordance with the Preferred Reporting Items for Systematic Reviews and MetaAnalyses. ${ }^{7}$ The protocol was registered with the International Prospective Register of Systematic Reviews (PROSPERO) ${ }^{8}$ Considering the date of the earliest confirmed reports of COVID-19, we have searched PubMed, Medline, Embase and Cumulative Index to Nursing and Allied Health Literature for studies published since 1 December 2019. The WHO database of publications on novel coronavirus was additionally searched for potentially relevant publications. ${ }^{9}$ We also searched the references listed within the retrieved articles to try and identify additional citations that may have been missed during the electronic search.

Eligible studies (including case reports, case series, cohort studies and retrospective studies) published in English-language peer-reviewed journals that have described original demographic and clinical characteristics of children diagnosed with COVID-19 were included for analysis. Review articles and opinion articles not reporting original data were excluded.

For the purpose of the electronic search, studies were identified with the following search terms: (coronavirus OR covid19 OR COVID-19 OR SARS-Cov-2 OR 2019-nCoV) AND (neonat* OR infant OR preterm OR premature OR Prem) AND (case report OR case series OR symptom OR symptoms OR characteristics OR characteristic*). We included both medical subject headings and free-text terms.

Two independent reviewers (MG and TK) evaluated the titles and abstracts of papers to identify relevant studies. Articles identified were then independently assessed by the two reviewers using predefined eligibility criteria. Any disagreements between authors regarding study inclusion were resolved through discussion among the reviewers.

\section{Risk of bias}

For cohort and case-control studies, we used the NewcastleOttawa tool $^{10}$ to assess the risk of bias. For case reports and case series, we used the tool suggested by Murad et al. ${ }^{11}$ This had previously been used in published systematic reviews, ${ }^{12}$ and was adapted from the Newcastle-Ottawa tool by the removal of items that relate to comparability and adjustment (which are not relevant to non-comparative studies) and retained items that focused on selection, representativeness of cases, and ascertainment of outcomes and exposure. This resulted in five criteria in the form of questions with a binary response (yes/no), whether the item was suggestive of bias or not. We considered the quality of the report as good (low risk of bias) when all five criteria were fulfilled, moderate when four were fulfilled, and poor (high risk of bias) when three or fewer were fulfilled. In the end, all the eight selected studies had a low risk of bias and were deemed good (table 1).

\section{Data extraction}

Data were extracted independently by the two reviewers on to a Microsoft Excel spreadsheet using a predefined checklist. Extracted data included but were not limited to the following: study design, year of publication, country, author name(s), number of patients, patient demographics, gastrointestinal symptoms and/or signs, other clinical symptoms and timing of gastrointestinal symptoms in relation to respiratory symptoms. The extracted data were compared and differences were discussed and resolved by consensus.

\section{Statistical analysis}

Statistical analyses were mainly descriptive (numerical and graphical). Frequencies and proportions were used to summarise qualitative variables, whereas means, medians, SD and quartiles or ranges were used to summarise quantitative variables. Where possible, meta-analyses

Table 1 Risk of bias assessment of the included studies (case reports and case series)

\begin{tabular}{|c|c|c|c|c|c|c|c|}
\hline Study & Cases (n) & Question 1 & Question 2 & Question 3 & Question 4 & Question 5 & Risk of bias \\
\hline Alzamora et a $\left.\right|^{29}$ & 1 & Yes & Yes & Yes & Yes & Yes & Low \\
\hline Kamali Aghdam et a/ ${ }^{30}$ & 1 & Yes & Yes & Yes & Yes & Yes & Low \\
\hline Chacón-Aguilar et a ${ }^{31}$ & 1 & Yes & Yes & Yes & Yes & Yes & Low \\
\hline Piersigilli et $a /^{32}$ & 1 & Yes & Yes & Yes & Yes & Yes & Low \\
\hline Wang et $a /^{33}$ & 1 & Yes & Yes & Yes & Yes & Yes & Low \\
\hline Wang et $a \beta^{34}$ & 1 & Yes & Yes & Yes & Yes & Yes & Low \\
\hline Yu et $a l^{35}$ & 1 & Yes & Yes & Yes & Yes & Yes & Low \\
\hline Zeng et $a l^{36}$ & 3 & Yes & Yes & Yes & Yes & Yes & Low \\
\hline
\end{tabular}

Tool details

Low risk items: Green shading, Unclear risk: Yellow shading, High risk: red shading 
using the random-effects model were performed to assess associations between demographic features and gastrointestinal symptoms. All statistical analyses were performed using StatsDirect statistical software.

\section{Case report}

The research and development department approved the writing of this paper.

\section{Patient and public involvement}

Patients and/or the public were not involved in the design, or conduct, or reporting, or dissemination plans of this research.

\section{RESULTS}

Our search was performed on 12 May 2020, and initial screening yielded 27 publications for full-text searching when screened against the inclusion and exclusion criteria. One of these publications was unrelated to neonates and was excluded. ${ }^{13}$ Ten other publications were excluded as the neonate(s) in all these were not confirmed to be infected. ${ }^{14-24}$ It is worth noting that one of the cases ${ }^{24}$ reported a COVID-19-positive mother who was very unwell and delivered by caesarean section at the bedside; the 35-week neonate unfortunately died within
2 hours. No further details regarding the neonate are described and confirmation of diagnosis is not reported; hence, this case was excluded.

Four other publications were excluded as these were review articles (figure 1). ${ }^{25-28}$

This left eight articles that described neonates with COVID-19 confirmed on reverse transcription (RT)PCR swabs. The characteristics of the included studies are shown in table 2. They included descriptions of one neonate each from Peru, ${ }^{29}$ Iran, ${ }^{30}$ Spain $^{31}$ and Belgium ${ }^{32}$ and six from China. ${ }^{33-36}$

The clinical characteristics of the 10 babies described in the eight publications are listed in table 2.

The report from Peru ${ }^{29}$ describes the occurrence of COVID-19 in a presumed 34-week baby who tested positive as early as 16 hours of life. The baby was intubated on day 0 and needed respiratory support for 6 days, but made an uneventful recovery therefrom. Imaging was negative. The baby had been separated from the mother at birth and had no postnatal contact with the family or anyone who was coronavirus-positive. This may be a case of vertical transmission.

The Iranian case report ${ }^{30}$ describes the occurrence of COVID-19 in a 15-day-old neonate. Details regarding the neonate's delivery records are sketchy. This is unlikely

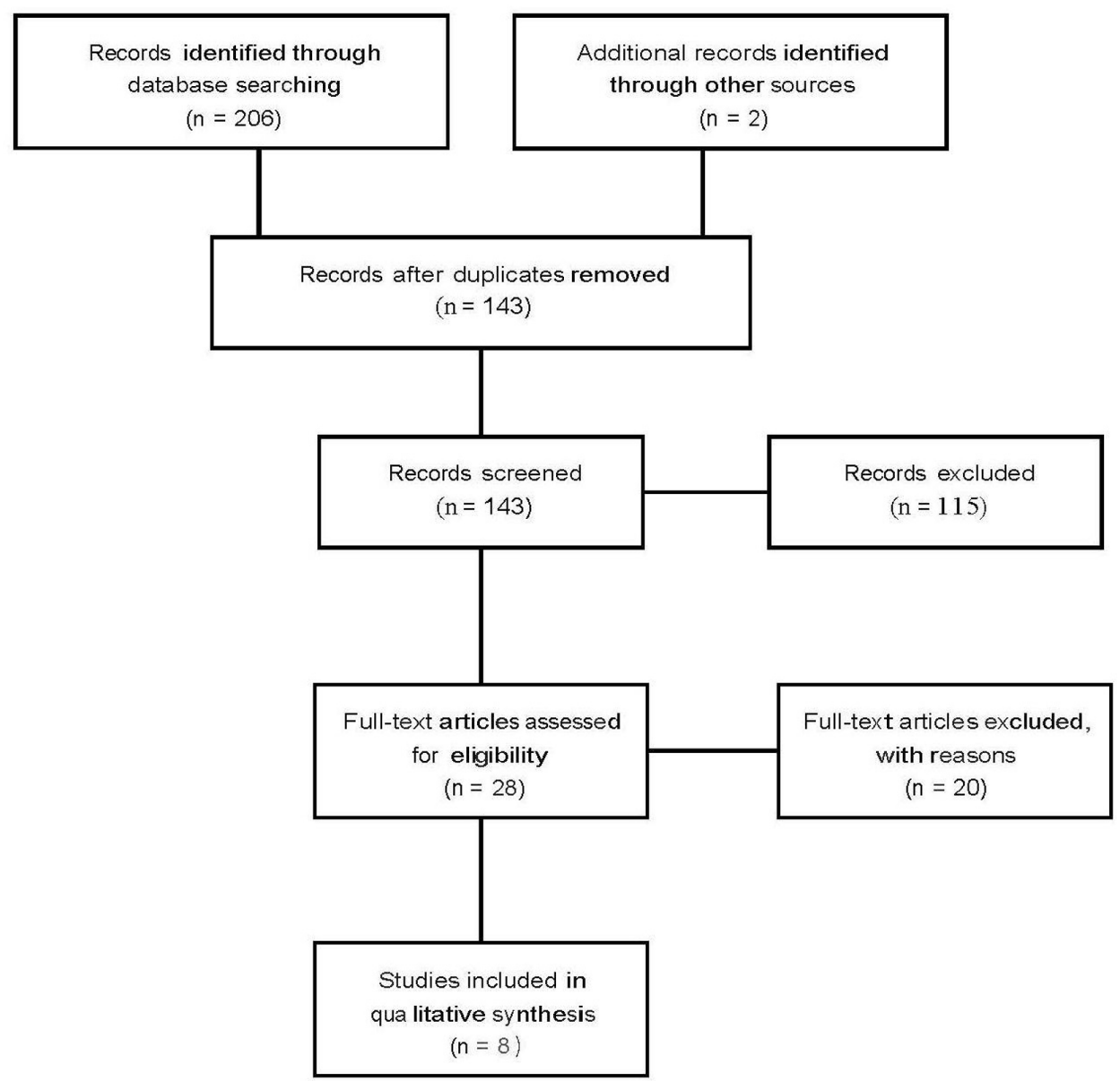

Figure 1 Flow diagram for study selection. 


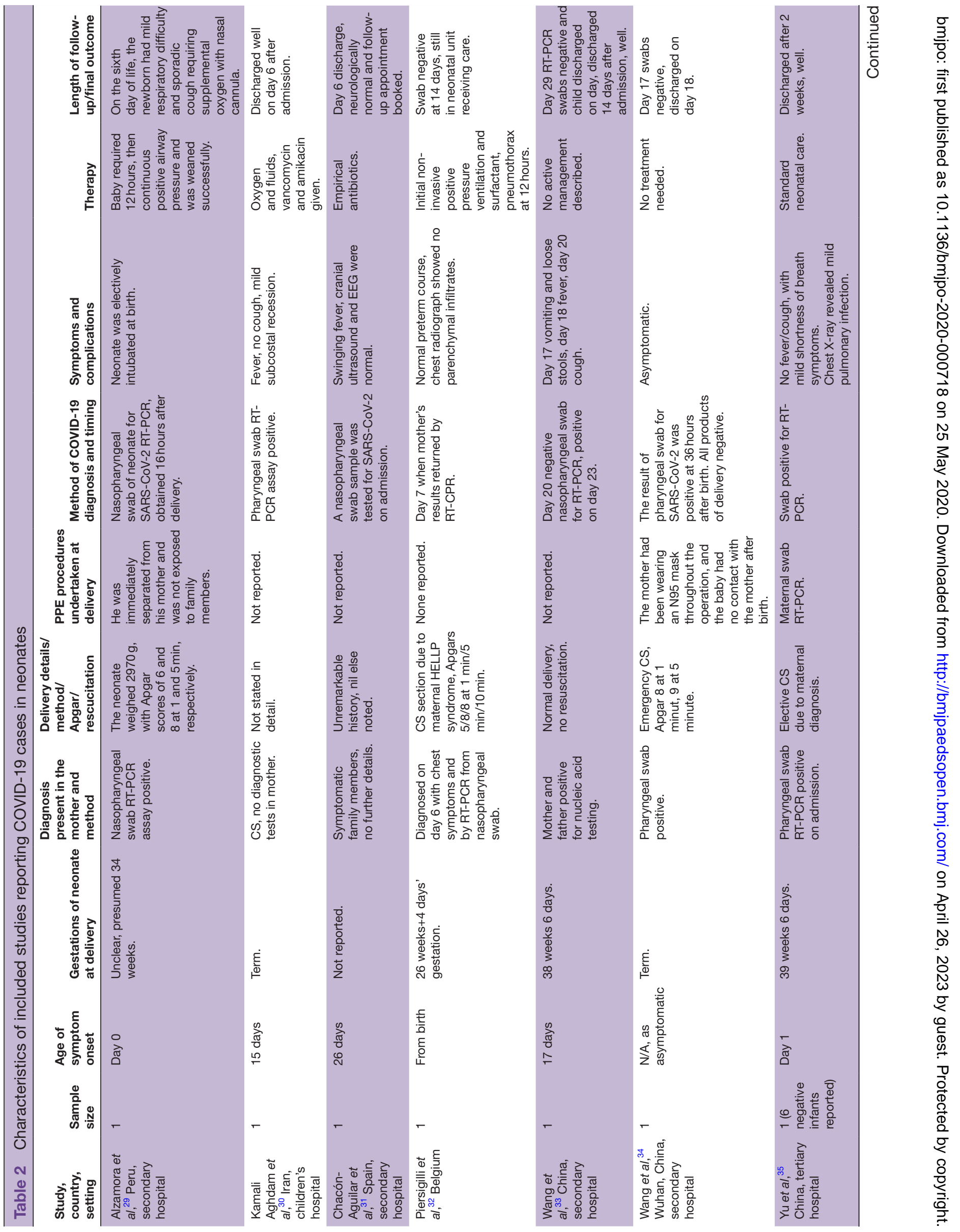


to be a vertical transmission. Also, the mother did not undergo any diagnostic tests.

The report from Spain ${ }^{31}$ describes a 26-day-old neonate presenting to the hospital with two episodes of presumed seizures. The baby was treated empirically with antibiotics, but tested positive for SARS-CoV-2 on nasopharyngeal swab. The baby was discharged after 6 days, with recommendations for home isolation for a further period of time. He was clinically well at discharge.

The report from Belgium ${ }^{32}$ is of a baby delivered at $26+4$ weeks of gestation and discovered to have a positive nasopharyngeal swab for SARS-CoV-2 RT-PCR done on her after her mother tested positive on account of ill health on day 7 after preterm delivery by caesarean section. The baby's results turned negative 14 days after the initial positive result. She was still on the neonatal unit on account of her extreme prematurity.

The four case reports from China are from secondary (2) and tertiary (2) hospitals. In the study by Wang et $a l,{ }^{33}$ both parents were COVID-19-positive. The baby presented with vomiting and diarrhoea on day 17 of life and was initially negative up to the 20th day of life, but turned positive on day 23, and later negative on day 29. The baby was discharged 14 days after admission and remained well, with no respiratory symptoms and needing no treatment.

The case described by Wang et $a l^{34}$ is a presumed vertical transmission in a term neonate that tested positive at 36 hours after birth. Placental and birth products tested negative, and the baby and the mother had no contact intranatally or postnatally. The baby remained asymptomatic and was discharged on day 18 after the 17th-day swabs tested negative.

The report by $\mathrm{Yu}$ et $a l^{5}$ describes a COVID-19-positive asymptomatic term neonate born to a positive mother by caesarean section. The baby was discharged after 2 weeks' stay in Wuhan Children's Hospital in Wuhan, China. This study reported the outcome in six other positive mothers whose babies tested negative.

Finally, Zeng et $a l^{36}$ describe 3 out of 33 neonates in their series from the Wuhan Children's Hospital who tested positive on nasopharyngeal swabs for SARS-CoV-2 by RT-PCR. All the neonates were born by caesarean section. In each case, the mother had COVID-19 pneumonia. While two babies were born at term, one was born preterm. All babies were symptomatic and had positive swabs on days 2 and 4 of life, but tested negative on days 6 (in two babies) and 7 (in the preterm baby).

\section{Case report}

A 36-year-old, gravida 3 mother presented at 32 weeks of gestational age with cough, high fever, lymphopaenia and a positive RT-PCR swab for SARS-CoV-2. She underwent an emergency caesarean section after being given two doses of antenatal steroids 12 hours apart. The mother wore a fluid-resistant mask, and the entire operative team was dressed in full personal protective equipment. The baby was a live, female newborn weighing 2150 g. She 
was directly handed over to the neonatal team without any contact with the mother. Postnatally, she has had no contact with the mother or the father or any family member.

She needed respiratory support, increasing from highflow nasal oxygen and then requiring surfactant via less invasive surfactant administration, progressing to intubation. The baby was extubated the next day and then moved to biphasic positive airway pressure support, weaning over the next few days to air.

The radiograph showed findings consistent with surfactant deficiency lung disease. The initial RT-PCR swab on day 1 of life was negative, but the child had a positive swab on day 4, day 14 and also on day 21 , while being asymptomatic.

While the baby gradually recovered and is being isolated in the neonatal unit and being fed and nursed, the mother deteriorated rapidly after the delivery and was kept on a ventilator in the intensive care unit of the hospital for a week. She underwent tracheostomy. Over the past week, she continued to improve, and she has now been moved out of the intensive care unit and the tracheostomy has been closed. She remains on oxygen at the time of writing this paper.

The baby remained RT-PCR-positive on day 28 of life, but was clinically well, and was discharged with the mother on day 33 of life.

\section{DISCUSSION}

This systematic review of published literature has shown only 10 case reports of neonatal COVID-19 infection. While seven of these ten babies were full-term, three were preterm, the lowest being at $26+4$ weeks of gestation. Three are suspected to be vertically transmitted, in addition to our own case, the summary of which has been described. Ours is the first case of a premature baby with COVID-19 in the UK and the fourth preterm baby globally. Also, our baby continued to remain positive at 28 days of life. It remains to be seen when the baby will become RT-PCR-negative, although it is clear that the baby is otherwise asymptomatic from the coronavirus infection.

Global knowledge about this new infectious pandemic is evolving. The current knowledge is that the virus is less virulent in children, although there have been case reports of older children dying as a result of contracting the infection. There is no available proven treatment, although many trials on different known and new drugs are ongoing both in the UK and elsewhere in the world.

Our rapid review concentrates on neonatal COVID19. The methodology was performed rapidly; however, this did not impact the high-quality systematic approach that such a review requires. We present only 10 positive neonatal cases from the global literature within the last 5 months since the pandemic started to emerge, and only three were potentially of vertical transmission. Within our excluded studies, it is worth noting that a further 65 neonates were reported as born to COVID-19-positive mothers who themselves tested negative on RT-PCR. ${ }^{1416-22}$ As the goal of the review was not to report well neonates born to infected mothers, this is likely a small representation of a much larger sample of reported cases of well neonates. Therefore, it does currently seem reasonable to suggest that vertical transmission to neonates and neonatal infection from infected family members are both rare occurrences. Additionally, when they do occur, the course appears mild. A previous review which was performed up to March 2020 considered children and neonates and found a similar mild course within these cohorts. ${ }^{37}$

The main limitation of this review is the relative novelty of COVID-19. It is possible that many more cases are either not reported to scholarly texts or in the process of peer review, and the reader must bear this in mind. Therefore, it is likely this review will be rapidly out of date and a repeat review will be required. Similarly, the scope of the review was very precise, and therefore it is inappropriate to suggest any firm conclusions regarding the incidence of COVID-19-negative neonatal cases born to infected mothers or the details of maternal outcomes, which would both have to be specific outcomes and are better covered in works focused on these issues, some of which were uncovered in this review. ${ }^{23} 25$

We believe that many other reports of this kind will emerge. It is key that future research considers the possibility of transmission from asymptomatic mothers. This will likely become of greater interest as population testing increases. It is also of particular interest that the case we report has remained RT-PCR-positive on day 28 and on discharge, despite being asymptomatic. Questions as to the time needed for seroconversion in infected children, the ability to spread while being asymptomatic and ongoing immunity after infection all require future investigation.

\section{CONCLUSIONS}

Neonatal infection has only been reported in 10 cases, with this study adding a case report of an eleventh case. Three occurred in the late neonatal period and three were presumed cases of vertical transmission, with the addition of the case we report likely to be of vertical transmission. All 10 previously reported cases had a mild course, and respiratory symptoms were generally consistent with their gestational age. Ongoing research is needed to ascertain the epidemiology of COVID-19 in neonates.

\section{Twitter Morris Gordon @drmorrisgordon and Taher Kagalwala @drtaher}

Acknowledgements The authors would like to thank the father of the baby for giving us the permission to publish the case report anonymously. Thanks are also due to the virology department of the hospital for reporting on the swabs of the baby.

Contributors MG ant TK led the systematic review and manuscript drafting. KR, $\mathrm{CR}, \mathrm{MIA}$ and $\mathrm{AG}$ cared for the neonate reported, contributed to the manuscript and approved the submission. 
Funding The authors have not declared a specific grant for this research from any funding agency in the public, commercial or not-for-profit sectors.

Competing interests None declared.

Patient consent for publication Next of kin consent obtained.

Provenance and peer review Not commissioned; externally peer reviewed.

Data availability statement All data relevant to the study are included in the article or uploaded as supplementary information.

Open access This is an open access article distributed in accordance with the Creative Commons Attribution Non Commercial (CC BY-NC 4.0) license, which permits others to distribute, remix, adapt, build upon this work non-commercially, and license their derivative works on different terms, provided the original work is properly cited, appropriate credit is given, any changes made indicated, and the use is non-commercial. See: http://creativecommons.org/licenses/by-nc/4.0/.

ORCID iDs

Morris Gordon http://orcid.org/0000-0002-1216-5158

Taher Kagalwala http://orcid.org/0000-0002-9846-9696

\section{REFERENCES}

1 Bedford J, Enria D, Giesecke J, et al. WHO Strategic and Technical Advisory Group for Infectious Hazards. COVID-19: towards controlling of a pandemic. Lancet 2020;S0140-6736:30673-5.

2 World Health Organization. Available: https://www.who.int/docs/ default-source/coronaviruse/situation-reports/20200512-covid-19sitrep-113.pdf?sfvrsn=feac3b6d 2

3 He F, Deng Y, Li W. Coronavirus disease 2019 (COVID-19): what we know? J Med Virol 2020.

4 Lai C-C, Liu YH, Wang C-Y, et al. Asymptomatic carrier state, acute respiratory disease, and pneumonia due to severe acute respiratory syndrome coronavirus 2 (SARS-CoV-2): facts and myths. J Microbiol Immunol Infect 2020:S1684-1182(20)30040-2.

5 Gu J, Han B, Wang J. COVID-19: gastrointestinal manifestations and potential Fecal-Oral transmission. Gastroenterology 2020;158:1518-9

6 Chan JF-W, Yuan S, Kok K-H, et al. A familial cluster of pneumonia associated with the 2019 novel coronavirus indicating personto-person transmission: a study of a family cluster. Lancet 2020;395:514-23.

7 Moher D, Liberati A, Tetzlaff J, et al. Preferred reporting items for systematic reviews and meta-analyses: the PRISMA statement. $J$ Clin Epidemiol 2009;62:1006-12.

8 University of Leicester. PROSPERO: International prospective register of systematic reviews. Available: https://www2.le.ac.uk/ library/find/databases/p/Prospero

9 World Health Organization. WHO database of publications on coronavirus disease (COVID-2019), 2020. Available: www.who.int/ emergencies/diseases/novel-coronavirus-2019/global-research-onnovel-coronavirus-2019-ncov

10 Newcastle - Ottawa quality assessment scale cohort studies. Available: http://www.evidencebasedpublichealth.de/download/ Newcastle_Ottawa_Scale_tool.pdf

11 Murad MH, Sultan S, Haffar S, et al. Methodological quality and synthesis of case series and case reports. BMJ Evid Based Med 2018;23:60-3

12 Bazerbachi F, Leise MD, Watt KD, et al. Systematic review of mixed cryoglobulinemia associated with hepatitis $E$ virus infection: association or causation? Gastroenterol Rep 2017:5:178-84

13 Wei M, Yuan J, Liu Y, et al. Novel coronavirus infection in hospitalized infants under 1 year of age in China. JAMA 2020:323:1313-4

$14 \mathrm{pp}$ Chen H, Guo J, Wang C, et al. Clinical characteristics and intrauterine vertical transmission potential of COVID-19 infection in nine pregnant women: a retrospective review of medical records. Lancet 2020;395:809-15.
15 Goldstein M. Chinese study suggests COVID-19 is not transmitted from pregnant mothers to newborns. Neonatol Today 2020:60-2.

16 Lowe B, Bopp B. COVID-19 vaginal delivery - a case report. Aust N Z J Obstet Gynaecol 2020. doi:10.1111/ajo.13173. [Epub ahead of print: 15 Apr 2020]

17 Liu W, Wang J, Li W, et al. Clinical characteristics of 19 neonates born to mothers with COVID-19. Front Med 2020;14:193-8.

18 Peng Z, Wang J, Mo Y, et al. Unlikely SARS-CoV-2 vertical transmission from mother to child: a case report. $J$ Infect Public Health 2020;13:818-20.

19 Chen Y, Peng H, Wang L, et al. Infants born to mothers with a new coronavirus (COVID-19). Front Pediatr 2020;8.

20 Zhu H, Wang L, Fang C, et al. Clinical analysis of 10 neonates born to mothers with 2019-nCoV pneumonia. Transl Pediatr 2020:9:51-60.

21 Li N, Han L, Peng M, et al. Maternal and neonatal outcomes of pregnant women with COVID-19 pneumonia: a case-control study. Clin Infect Dis 2020. doi:10.1093/cid/ciaa352. [Epub ahead of print: 30 Mar 2020]

22 Yang P, Wang X, Liu P, et al. Clinical characteristics and risk assessment of newborns born to mothers with COVID-19. J Clin Virol 2020;127:104356.

23 Liu D, Li L, Wu X, et al. Pregnancy and perinatal outcomes of women with coronavirus disease (COVID-19) pneumonia: a preliminary analysis. AJR Am J Roentgenol 2020:1-6.

$24 \mathrm{Li}$ J, Wang Y, Zeng Y, et al. Critically ill pregnant patient with COVID-19 and neonatal death within two hours of birth. Int $J$ Gynecol Obstet 2020.

25 Kleinwechter H, Laubner K. Coronavirus disease 2019 (COVID-19) and pregnancy: overview and report of the first German case with COVID-19 and gestational diabetes Diabetologe, 2020.

26 Panahi L, Amiri M, Pouy S, et al. Risks of novel coronavirus disease (COVID-19) in pregnancy; a narrative review. Arch Acad Emerg Med 2020;8:e34.

27 Zaigham M, Andersson O. Maternal and perinatal outcomes with COVID-19: a systematic review of 108 pregnancies. Acta Obstet Gynecol Scand 2020. doi:10.1111/aogs.13867. [Epub ahead of print: 07 Apr 2020].

28 Parazzini F, Bortolus R, Mauri PA, et al. Delivery in pregnant women infected with SARS-CoV-2: a fast review. Int J Gynaecol Obstet 2020. doi:10.1002/ijgo.13166. [Epub ahead of print: 09 Apr 2020].

29 Alzamora MC, Paredes T, Caceres D, et al. Severe COVID-19 during pregnancy and possible vertical transmission. Am J Perinatol 2020. doi:10.1055/s-0040-1710050. [Epub ahead of print: 18 Apr 2020]

30 Kamali Aghdam M, Jafari N, Eftekhari K. Novel coronavirus in a 15-day-old neonate with clinical signs of sepsis, a case report. Infect Dis 2020;52:427-9.

31 Chacón-Aguilar R, Osorio-Cámara JM, Sanjurjo-Jimenez I, et al. COVID-19: fever syndrome and neurological symptoms in a neonate. An Pediatr 2020. doi:10.1016/j.anpede.2020.04.001. [Epub ahead of print: 27 Apr 2020]

32 Piersigilli F, Carkeek K, Hocq C, et al. COVID-19 in a 26-week preterm neonate. Lancet Child Adolesc Health 2020. doi:10.1016/ S2352-4642(20)30140-1. [Epub ahead of print: 07 May 2020].

33 Wang J, Wang D, Chen G-C, et al. SARS-CoV-2 infection with gastrointestinal symptoms as the first manifestation in a neonate. Chi J of Contemp Ped 2020;22:211-4.

34 Wang S, Guo L, Zhang J, et al. A case report of neonatal COVID-19 infection in China. Clin Infec Dis 2020:ciaa225.

35 Yu N, Li W, Kang Q, et al. Clinical features and obstetric and neonatal outcomes of pregnant patients with COVID-19 in Wuhan, China: a retrospective, single-centre, descriptive study. Lancet Infect Dis 2020;20:559-64.

36 Zeng L, Xia S, Yuan W, et al. Neonatal early-onset infection with SARS-CoV-2 in 33 neonates born to mothers with COVID-19 in Wuhan, China. JAMA Pediatr 2020. doi:10.1001/ jamapediatrics.2020.0878. [Epub ahead of print: 26 Mar 2020].

37 Panahi L, Amiri M, Pouy S. Clinical characteristics of COVID-19 infection in newborns and pediatrics: a systematic review. Arch Acad Emerg Med 2020;8:e50. 